\title{
Teorías subjetivas de profesores sobre la motivación y sus expectativas de éxito y fracaso escolar ${ }^{1}$
}

\section{Teorias subjetivas de professores sobre a motivação e suas expectativas de sucesso e fracasso escolar}

\section{Subjective theories of teachers about motivation and their expectancies about school success and failure}

\author{
Pablo Javier Castro-Carrasco ${ }^{2}$ \\ Francis General ${ }^{3}$ \\ Richard Jofré ${ }^{4}$ \\ Nicolás Sáez ${ }^{5}$ \\ Ángela Vega ${ }^{6}$ \\ Mariella Bortoluzzi ${ }^{7}$
}

\begin{abstract}
RESUMEN
Investigación cualitativa que busca describir e interpretar las teorías subjetivas de un grupo de profesores de un liceo técnico público acerca de la
\end{abstract}

${ }^{1}$ Este estudio se encuentra inserto en el proyecto DIULS 220-2-07, titulado "Reflexión colectiva sistemática en el desempeño docente: un estudio orientado al desarrollo profesional". La Serena: Universidad de La Serena.

${ }^{2}$ Doctor en Psicología de la Pontificia Universidad Católica, Chile, Académico del Departamento de Psicología, Universidad de La Serena (ULS), Chile. E-mail: pablocastro@userena.cl

${ }^{3}$ Psicólogo, Universidad de La Serena (ULS), Chile. E-mail: framaury@hotmail.com

${ }^{4}$ Psicólogo, Universidad de La Serena (ULS), Chile. E-mail: indetestable@gmail.com

${ }^{5}$ Psicólogo, Universidad de La Serena (ULS), Chile. E-mail: nsp_86@msn.com

${ }^{6}$ Psicóloga, Universidad de La Serena (ULS), Chile. E-mail: angelavegap@gmail.com

${ }^{7}$ Magister (C) en Psicología, mención Psicología Educacional, de la Universidad de La Serena, Chile. E-mail: maribortoluzzi@gmail.com 
motivación escolar (rol como agente motivacional y motivación inherente a las alumnas) y sus expectativas acerca del éxito y fracaso escolar. Los datos recolectados mediante observación directa de reuniones de profesores y de una entrevista grupal en profundidad, fueron analizados mediante el programa Atlas-Ti, siguiendo orientaciones de la Grounded Theory. Los resultados muestran que la sobreatribución que hacen los profesores acerca de su responsabilidad como agente motivacional ignora otras variables del proceso enseñanza-aprendizaje, dependientes tanto de las alumnas como del contexto educativo, y que sus expectativas de éxito o fracaso se relacionan directamente con los objetivos del establecimiento.

Palabras-clave: teorías subjetivas; motivación; expectativas del profesor.

\title{
RESUMO
}

Pesquisa qualitativa que procura descrever e interpretar as teorias subjetivas de um grupo de professores de uma escola técnica pública sobre a motivação escolar (seu papel como agente motivacional e motivação relacionada às alunas) e suas expectativas a respeito do sucesso e fracasso escolar. Os dados coletados, mediante observação direta de reuniões dos professores e de uma entrevista grupal em profundidade, foram analisados utilizando-se o programa Atlas-Ti, seguindo as orientações da Grounded Theory. Os resultados mostram que a supervalorização, por parte dos professores, quanto à sua responsabilidade como agente motivacional ignora outras variáveis no processo ensino-aprendizagem, dependentes tanto das alunas como do contexto educativo, e que suas expectativas de sucesso ou fracasso se relacionam diretamente com os objetivos do estabelecimento.

Palavras-chave: teorias subjetivas; motivação; expectativas do professor.

\begin{abstract}
Qualitative research that looks to describe and to interpret the subjective theories of a group of teachers from a public technical high school about school motivation (its role as a motivational agent and the motivation inherent in the pupils), and their expectances about the school success and school failure. The information gathered by means of direct observation of meetings of teachers and of interviews in depth was analyzed by means of the program Atlas-Ti, following orientations from the Grounded Theory. The results show that the over attribution that the teachers do about their responsibility as motivational agents, ignores other variables of the teachinglearning process. It depends so much on the pupils as on the educational context, and that their success-failure expectancies are related directly to the targets of the establishment.
\end{abstract}

Keywords: subjective theories; motivation; teacher expectancies. 


\section{Introducción}

En la actualidad, el lineamiento por el que se guían las nuevas concepciones de enseñanza, ha experimentado un cambio importante que pone énfasis en la reflexión del profesor acerca de su práctica pedagógica y sus implicancias en la motivación para el aprendizaje escolar de los estudiantes.

En particular concordamos con Sepúlveda, Reyes y Pérez (2003), respecto a que se requiere la realización de estudios que tengan como finalidad comprender qué determina la motivación para el aprendizaje de los estudiantes. Desde la perspectiva de este estudio, parte de esa comprensión está relacionada con abordar las propias explicaciones de los docentes respecto a la motivación escolar, el rol que ejercen en ella y su relación el éxito y fracaso de sus estudiantes.

Es por tal razón, que el presente estudio se centra en las explicaciones de los profesores acerca de la influencia que ejercen en el aprendizaje de sus alumnas, específicamente mediante su propio rol como agente motivacional, y en las expectativas de resultado (BANDURA, 1999), de éxito o fracaso, que poseen en relación a estas.

La presente investigación tuvo como objetivo describir e interpretar las teorías subjetivas sustentadas por un grupo de profesores de enseñanza media de un liceo técnico profesional acerca de la motivación escolar (su rol como agentes motivacionales y la motivación de las alumnas orientada hacia el aprendizaje), además de sus expectativas acerca del éxito y fracaso escolar.

\section{Teorías subjetivas}

Las teorías subjetivas (TS) o implícitas, como objeto de estudio, se definen como un tipo particular de creencia de carácter fundamentalmente explicativo (supuestos o hipótesis), que sustentan las personas, acerca de ellos mismos y de su contexto (DWECK, 1995, FLICK, 1993, GOPNIK, 1996; GROEBEN, 1990; KRAUSE, 2005, GROEBEN; SCHEELE, citados en GÜRTLER, 2001). Esto les posibilita además de comprender su propio mundo, comunicarse con el mismo en general. En este sentido, las TS tienen una influencia sobre la forma como el profesional comprende, planifica y posteriormente actúa en su quehacer (FLICK, 2004).

Tal como plantean Karmilloff-Smith y Inhelder (1974), las teorías personales tienen un efecto regulador de las acciones, en este caso del docente, 
comprendido este efecto, como la composición de los esquemas mentales influyendo en el actuar (FLICK, 2004). Si asumimos a las teorías subjetivas (o implícitas) como "modelos dinámicos de conocimiento" (RODRIGO; RODRÍGUEZ; MARRERO, 1993, p. 104), podríamos dar cuenta de las diversas variaciones que pueden tener las teorías subjetivas en el sujeto a lo largo de su desarrollo (personal y profesional). Desde esta perspectiva, una teoría subjetiva puede tener cambios, transformaciones, crecimientos o reducciones a lo largo del tiempo. Así, el cambio de las teorías subjetivas, según Krause (2005) se produce mediante un proceso secuencial, determinado en fases y con momentos (CASTRO, 2008).

En particular este trabajo, sin bien no reporta el análisis del cambio de TS, está enmarcado en una investigación mayor, estructurada en diferentes fases de análisis. ${ }^{8}$ Así, el estudio reportado aquí busca comprender las TS de docentes de un establecimiento educacional técnico acerca de la motivación y expectativas de éxito y fracaso de sus alumnas, durante la fase definida como el "antes", previo a la realización de una intervención en estrategias de reflexión colectiva sistemática con este grupo de docentes (CATALÁN; CASTRO, 2008). Por tanto, los presentes hallazgos han constituido un insumo para las posteriores fases del proyecto de investigación, en específico, para el análisis del cambio de TS en los profesores.

\section{Motivación escolar, una llave para el aprendizaje}

El constructo motivación ha sido explicado desde distintas concepciones, con teorías de carácter biológico o fisiológicas, algunas humanistas, hasta las más recientes teorías cognitivas (SEPÚLVEDA; REYES; PÉREZ, 2003).

Parece fundamental, en este punto, destacar la importancia que han tenido algunos autores al contribuir con explicaciones acerca del fenómeno de la motivación, entre ellos, se destacan los aportes de autores como Atkinson (1964) y su teoría sobre la motivación de logro, la cual se entiende como "[...] una tendencia a conseguir una buena ejecución (éxito) en situaciones que implican competición con una norma, como un estándar de excelencia, siendo la ejecución evaluada como éxito o fracaso, por el propio sujeto o por otros" (GARRIDO, 1986, p. 138) y la teoría atribucional de la motivación y la emoción, de Weiner (1986a,

${ }^{8}$ Proyecto DIULS 220-2-07, titulado "Reflexión colectiva sistemática en el desempeño docente: Un estudio orientado al desarrollo profesional”. La Serena: Universidad de La Serena. 
1986b), cuya idea fundamental es que las atribuciones causales efectuadas por un individuo condicionan sus expectativas futuras y sus sentimientos, y ambos, expectativas y emociones, condicionan la acción.

En la motivación escolar se interrelacionan diversos componentes cognitivos, afectivos, sociales y académicos que tienen que ver tanto con las acciones de los alumnos como con la de sus profesores. La motivación se encuentra presente por tanto, en todo acto de enseñanza-aprendizaje, conducta que puede ser regulada de forma intrínseca o extrínseca. Siendo la motivación extrínseca la que está dirigida hacia recompensas externas y la motivación intrínseca aquella que busca satisfacer necesidades psicológicas tales como la autodeterminación y el sentido de competencia (DECI; KOESTNER; RYAN, 1999).

\section{Atribuciones, formadoras de expectativas}

Considerando que la motivación se define usualmente como algo "que activa, dirige y mantiene la conducta" (WOOLFOLK, 1996, p. 330), en el contexto escolar ésta posee dos dimensiones interrelacionadas que inciden directamente en el proceso enseñanza aprendizaje.

La primera dimensión es aquella relacionada con el profesor, en la que sus motivaciones guían las acciones de éste en el aula, y se relacionan directamente, con las expectativas que posee acerca de la capacidad de aprendizaje de sus alumnos. La segunda dimensión conformada por los alumnos, dice relación con su tipo de motivación, la cual puede o no estar orientada al aprendizaje.

Ambas dimensiones se relacionan en cuanto las expectativas sustentadas por los profesores acerca de las capacidades de los alumnos para aprender, interfieren tanto en su propia motivación para enseñar, como en la motivación de los alumnos por aprender.

Es así como al interior del aula cobran relevancia aspectos como la motivación para el aprendizaje y las expectativas del profesorado, por cuanto ambas se influyen (SEPÚLVEDA; REYES; PÉREZ, 2003).

Ahora bien, las expectativas son conformadas principalmente mediante procesos atribucionales, entendiendo las atribuciones en términos generales, como la forma en que las personas explican las conductas tanto de sí mismas como de los demás. Es así como la forma en que los profesores explican tanto sus acciones como la de sus alumnos (sus teorías subjetivas al respecto), genera expectativas que pueden ser de éxito o fracaso, y éstas por consiguiente, condicionan sus acciones pedagógicas comprometiendo su motivación en la ejecución de ellas. 


\section{Metodología}

Diseño

Esta investigación posee un diseño del tipo estudio de caso de carácter descriptivo-interpretativo.

\section{Participantes}

La muestra constó de 37 profesores de un tradicional liceo técnico municipal (público) de la ciudad de La Serena, Chile, de los cuales 8 participaron de una entrevista grupal y los 29 restantes fueron observados en reuniones de trabajo semanales durante un periodo de 3 meses.

\section{Fuentes de datos}

Se utilizaron dos fuentes de datos disponibles para el análisis presentado en este estudio: la fuente principal es un compilado de registros audiovisuales producto de la observación participante de las reuniones de trabajo de los profesores y una entrevista grupal realizada por otro investigador, para otro estudio sobre teoría subjetivas de docentes (RETUERT; CASTRO, 2011), con 8 profesores del mismo establecimiento, la que se uso como recurso de triangulación de datos (DENZIN, 1989).

\section{Análisis de datos}

Para analizar los datos nos basamos en los procedimientos sugeridos por la Grounded Theory de Glaser y Strauss (1967), que se define como la elaboración de una teoría, de forma inductiva, para la comprensión de un fenómeno particular del que se da cuenta, mediante procesos de codificación e interpretación (FLICK, 2004).

Las fases del análisis fueron: codificación abierta de los registros audiovisuales disponibles (sesiones y entrevista grupal), establecimiento de familias de códigos surgidos de la codificación, realización de una descripción de las familias de códigos y finalmente la modelización de los hallazgos. 


\section{Resultados}

A lo largo de la investigación, se describieron e interpretaron las teorías subjetivas que sustentaba un grupo de profesores acerca de la motivación escolar, además de sus expectativas acerca del éxito y fracaso escolar. Todo lo anterior bajo la naturaleza de estas concepciones en términos de condiciones, acciones y metas. ${ }^{9}$

Los hallazgos del presente estudio ponen en evidencia la existencia de condiciones específicas que caracterizan un tipo particular de teorías subjetivas, referidas tanto a la motivación en el proceso de enseñanza-aprendizaje, como también a las expectativas de éxito o fracaso escolar sustentadas por los profesores en un contexto educativo técnico.

Los resultados se encuentran apartados en dos dimensiones complementarias en su relación. La primera de ellas refiere a las teorías subjetivas que sustenta el grupo de profesores acerca de su propio rol como agente motivacional para el aprendizaje escolar; y la segunda dimensión representa las teorías subjetivas de estos profesionales sobre la motivación inherente que poseen sus alumnas hacia el aprendizaje (Figura 1).

En relación a los resultados definidos en la primera dimensión, resaltan las creencias que enfatizan un compromiso con el cumplimiento del rol que se asignan a sí mismos como agentes motivadores del aprendizaje escolar (locus de control interno).

"[...] en cuanto a los desafios en el aula, tenemos desafios como profesores, tener clases motivadoras como decíamos, estar dictando, hablándoles oralmente. Las niñas se aburren definitivamente" (Sesión 2, audio 4).

El cumplimiento del rol de agente motivacional, es descrito mediante funciones tales como: el desarrollo de clases con mayor contenido didáctico, donde se infiere el componente de "entretener para aprender"; además de la utilización de incentivos (reforzadores positivos) y el apoyo a las alumnas con mayores dificultades para el aprendizaje.

\footnotetext{
${ }^{9}$ En la descripción de los resultados y en las conclusiones la palabras o frases en cursiva corresponden a los conceptos usados por nosotros en el Modelo explicativo (Figura 1), para las frases textuales de los profesores usamos comillas y cursivas ( "”).
} 
"En cuanto a los procesos preventivos para crear un ambiente propicio para el aprendizaje, estarían [...] el incentivo con un puntaje que incida en la nota final del trabajo correspondiente, o sea que darle puntaje a cada cosa, yo con las niñas conversé y la que trae el libro de texto va a tener un punto para la prueba y la que no lo trae se le va a quitar, de alguna manera tratar de que ellas cumplan, al menos con el incentivo" (Entrevista grupal).

Por otro lado el incumplimiento de este rol, es atribuido a la percepción de una falta de motivación que poseen las alumnas hacia el aprendizaje (locus de control externo).

Es evidente en esta investigación que las expectativas del profesor acerca del éxito o fracaso escolar de sus alumnas influyen tanto en su rol como agente motivacional, y condiciona la capacidad de motivación de las propias alumnas. Si bien, la formación de estas expectativas es normal y no posee una valencia positiva o negativa en sí misma, estas afectan en la percepción e interpretación del cumplimiento o incumplimiento del rol motivacional que se asignan.

De esta manera se observa que en la situación de cumplimiento, los profesores están más prontos a percibir el éxito de logro de aprendizaje escolar, como producto directo de sus esfuerzos como agentes motivacionales. En cambio el incumplimiento, desarrolla una percepción de fracaso del logro focalizado en el desempeño motivacional exclusivo de sus estudiantes, restando o anulando su participación como factor en tales resultados.

Cabe mencionar que los profesores creen que las alumnas tienen motivación, pero que esta se encuentra orientada a otros fines distintos del aprendizaje, por tanto su rol es descrito como canalizador de la motivación hacia esa meta. Pero es en esta dimensión, donde se aprecian y describen variables que influencian fuertemente su desarrollo; una de ellas son las metodologías ineficientes que detecta este grupo de profesionales para motivar a sus alumnas, expresan conscientemente la necesidad de utilizar nuevas estrategias de enseñanza, sin embargo, éstas no se llevan a la práctica.

Otras variables son el contexto educativo donde desempeñan su profesión, señalando la influencia de su Proyecto Educativo Institucional (PEI), la condición de ser un Liceo Técnico Profesional y las características socioculturales de las propias estudiantes, donde se describe la precariedad y falencia valórica y afectiva como característico del alumnado.

\footnotetext{
"[...] nosotros sabemos y está de más decir, que nuestras alumnas provienen de hogares que tienen bastantes falencias, sobre todo en lo
} 
socio afectivo, entonces ahi se generan bastantes problemas, los cuales las niñas traen al aula y eso es lógico porque aún como están en el periodo de adolescencia, ellas no saben enfocar bien las problemáticas que ellas tienen.

En cuanto a la formación valórica y disciplinaria de la alumna [...] parte desde la perspectiva familiar [...] las alumnas no están en un ambiente propicio para aprender valores, entonces los vienen a aprender acá, $y$ eso también juega un papel preponderante que nosotros tenemos en el aula" (Entrevista grupal).

Además se señalan ciertas características personales, tales como la falta de interés en los contenidos escolares, la motivación orientada a otros fines y la relación entre la baja motivación con la "baja autoestima" que poseerían las estudiantes.

"[...] elevar el autoestima de las alumnas [...] es bastante complejo, las alumnas tienen el autoestima muy baja, lo que las lleva a no esforzarse, a no luchar con algo, a no sentirse motivadas" (Video 7, reunión 3).

"Basta que les cambie una palabra y no entiendan, hay que enseñarles tal cual las cosas, se quedan con lo que aprenden en la sala" (Video 2, sesión 2).

El uso de estrategias motivacionales utilizadas por este grupo de profesionales inciden en las expectativas de éxito o fracaso que sustentan; si éstas estrategias fallan el profesor entiende que ha incumplido su rol, o bien, que sus alumnas no son capaces de aprender, generándoles ambas situaciones ciertos grados de frustración.

Cabe señalar que estos profesores reconocen la existencia de alumnas motivadas hacia el aprendizaje, específicamente aquellas de la especialidad de secretariado,

"[...] No te olvides que las alumnas de secretariado, es la alumna que más estudia [...] ella ve otra posibilidad de seguir estudiando [...]", (Video 6, reunión 3).

Sin embargo, consideran que su motivación no es influenciada por el rol 


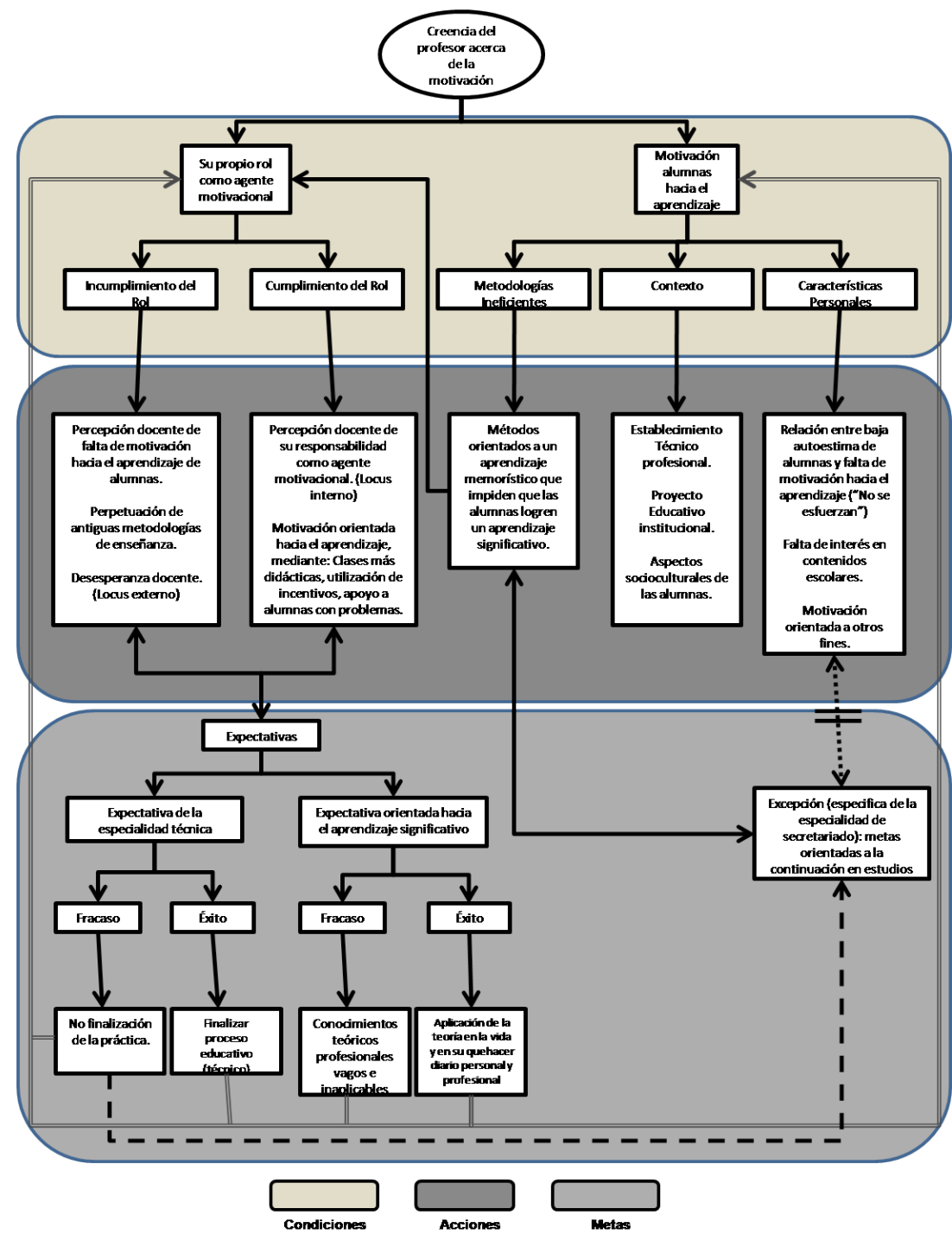

FIGURA 1 - Modelo explicativo: Teorías subjetivas acerca del rol del docente como agente motivacional y sobre la motivación de las alumnas hacia el aprendizaje. ${ }^{10}$

${ }^{10}$ Simbología utilizada en la figura: flecha unidireccional: influye, flecha bidireccional: influye reciprocamente, flecha segmentada: atribuye, flecha segmentada bidireccional intersecada por dos barras: diferentes. 
reconocido de agentes motivacionales. Es en este aspecto donde se aprecia un fenómeno particular en las teorías subjetivas que sustenta este grupo profesional, puesto que si bien reconocen la motivación de estas alumnas hacia el aprendizaje, las expectativas de fracaso que sostienen en base al carácter técnico profesional del establecimiento, son contradictorias en la interpretación. Ya que el fracaso que ellos sostienen en esta cualidad, es la no finalización de la práctica profesional; pero es en este grupo particular de alumnas de secretariado donde describen una alta deserción de sus prácticas, ya que ellos señalan que estas estudiantes tienen posibilidades y metas de continuación de sus estudios.

Las expectativas de fracaso que sostienen ante el aprendizaje significativo de sus alumnas, tienen relación con la adquisición de conocimientos teóricos profesionales vagos e inaplicables. En cuanto a las expectativas de éxito en este mismo ámbito, éstas están referidas a que las estudiantes logren la aplicación de estos conocimientos en su entorno y cotidianidad.

\section{Conclusiones}

En esta investigación se buscó describir e interpretar las teorías subjetivas de un grupo de profesores de un liceo técnico acerca de la motivación escolar, tanto desde su rol como agente motivacional como de sus creencias acerca de la motivación orientada al aprendizaje que poseen de manera inherente las alumnas. Así mismo, describir las expectativas sustentadas por ellos en relación al éxito y fracaso escolar de sus estudiantes.

En los resultados es posible distinguir que las TS del profesor acerca de la motivación, destacan fundamentalmente el rol que se asignan a sí mismos como agentes motivadores del aprendizaje escolar. Además, sus expectativas de éxito relacionadas con el cumplimiento de los objetivos planteados en el establecimiento escolar, se incrementan al estar conscientes del logro de ese rol de manera satisfactoria.

El uso de estrategias motivacionales entonces, inciden en las creencias de éxito o fracaso sustentadas por el profesor, si éstas fallan el profesor cree que ha incumplido su rol o bien, que sus alumnas no son capaces de aprender, y en ambos casos se frustra.

Ahora bien, a pesar de que el profesor está consciente de la necesidad de utilizar nuevas estrategias motivacionales de enseñanza, éstas no son llevadas a la práctica. 
Otro aspecto importante en relación a las expectativas del profesor acerca del éxito o fracaso de sus alumnas, y de cómo éstas influyen tanto en su rol como agente motivacional como en la motivación de las propias alumnas, es que si bien, el profesor cree que las alumnas tienen motivación, considera que está orientada a otros fines distintos del aprendizaje, y que por tanto, su rol debe ser el de encauzar esa motivación hacia el aprendizaje.

Por otra parte el hallazgo relativo a que los docentes perciben más el éxito que los fracasos de las estudiantes, es coincidente con los hallazgos acerca de la teoría de la atribución, en cuanto a que las expectativas que sustenta este grupo de profesionales, tienden a reforzarse a sí mismas, es decir, los profesores están más alertas a percibir lo que ellos esperan de la conducta de sus alumnas y tienen menos probabilidades de observar lo que no esperan, y cuando interpretan los hechos, esta interpretación es consistente con sus expectativas manifiestas.

Es posible ver también, que los profesores reconocen la existencia de un grupo de alumnas motivadas hacia el aprendizaje, específicamente aquellas de la especialidad de secretariado, sin embargo, además de considerarlas una excepción, creen la motivación de ellas no es influenciada por el ejercicio de su rol como agente motivacional.

Lo anterior, junto a la sobreatribución que hacen los profesores acerca de su responsabilidad como agente motivacional (atribución interna), que ignora otras variables del proceso enseñanza-aprendizaje, hacen pensar en la presencia en este grupo de docentes del hecho conocido como atribuciones "contra defensivas", es decir, el asumir la responsabilidad de los fracasos de los estudiantes, pero no la de sus éxitos (CLARK; PETERSON, 1990).

Además, la contradicción evidenciada por los profesores, entre el "saber qué hacer" y lo que "se hace" en términos de metodologías de enseñanza señala un conflicto fundamental en el ejercicio del rol de profesor y por lo tanto en las acciones que realiza para lograrlo.

Las expectativas de éxito o fracaso de los profesores se relacionan directamente con los objetivos del establecimiento, esto es, la realización de la práctica y posterior obtención de la especialidad técnica; ignorando los intereses de las alumnas más motivadas hacia la continuación de sus estudios, debido a que éstas no cumplen con las expectativas de éxito de los profesores.

En este sentido, este conjunto de teorías subjetivas, podrían estar condenando a este grupo de estudiantes a metas y/u objetivos escolares más modestos; en tanto no motivarían o incentivarían a este grupo a la meta orientada a la continuación de estudios posteriores, más bien están relacionadas a cumplir la expectativa de éxito que es finalizar el proceso educativo técnico y no la continuación de estudios superiores (en Chile, universidad, instituto profesional o centro de formación técnica). 
En síntesis, al parecer la meta buscada por los profesores parece determinar a las alumnas con mayor motivación intrínseca a la obtención de objetivos escolares limitados.

\section{REFERENCIAS}

ARANCIBIA, V.; ÁLVAREZ, M. I. Modelo de variables del profesor y su impacto en rendimiento escolar y autoconcepto académico. Proyecto Fondecyt N ${ }^{\circ} 1880405$. Santiago de Chile: Pontificia Universidad Católica de Chile, 1991.

ATKINSON, J. W. An Introduction to Motivation. Princeton, N. J.: Van Nostrand, 1964. AVENDAÑO, C.; KRAUSE, M.; WINKLER, M. I. Representaciones sociales y teorías subjetivas: relevancia teórica y aplicaciones empíricas. Psykhe, v. 2, n. 1, p. 107-114, 1993.

BANDURA, A. Auto-eficacia: cómo afrontamos los cambios en la sociedad actual. España: Desclée De Brouwer, 1999.

CASTRO, P. Cambio de teorías subjetivas en profesores respecto a la enseñanza y el aprendizaje de valores. Tesis (Doctorado en Psicología) - Pontificia Universidad Católica de Chile. Santiago, 2008.

CATALÁN, J. Del pensamiento al conocimiento profesional del profesor. En:

(Ed.). Psicología Educacional: proponiendo rumbos, problemáticas y aportaciones. Chile: Editorial Universidad de La Serena, 2011. p. 187-215.

; CASTRO, P. Proyecto DIULS 220-2-07, titulado "Reflexión colectiva sistemática en el desempeño docente: un estudio orientado al desarrollo profesional". La Serena: Universidad de La Serena, 2008.

CLARK, C.; PETERSON, P. Los procesos de pensamiento de los docentes. En: WITTROCK, M. C. La investigación de la enseñanza, III. Profesores y alumnos. Barcelona: Paidós-MEC, 1990. p. 443-539.

DECI, Edward L.; KOESTNER, Richard; RYAN, Richard M. A meta-analytic review of experiments examining the effects of extrinsic rewards on intrinsic motivation. Psychological Bulletin, v. 125, n. 6, p. 627-668, nov. 1999.

DENZIN, N. The research act (3ra ed.). Englewood Cliffs, N. J: Prentice Hall, 1989.

DWECK, C. S. Implicit theories as organizers of goals and behavior. En: GOLLWITZER, P. M.; BARGH, J. A. (Eds.). The psychology of action: linking cognition and motivation to behavior. New York: Guilford Press, 1995. p. 69-90. 
FLICK, U. (Ed.). La perception quotidienne de la santé et de la maladie. Théories subjectives et représentations sociales. Paris: L'Harmatan, 1993.

. Introducción a la investigación cualitativa. Madrid: Ediciones Morata, 2004.

GARRIDO, I. La motivación escolar: determinantes sociológicos y psicológicos del rendimiento. En: MAYOR, J. (Ed.). Sociología y Psicología de la Educación. Madrid: Anaya, 1986. p. 122-151.

GLASER, B.; STRAUSS, A. The Discovery of Grounded Theory: Strategies for Qualitative Research. Chicago: Aldine Press, 1967.

GOPNIK, A. The post-Piaget era. Psychological Science, v. 7, p. 221-225, 1996.

GROEBEN, N. Subjective theories and the explanation of human action. En: SEMIN, G. R.; GERGEN, K. (Eds.). Everyday understanding. Social and scientific implications. London: Sage, 1990.

GÜRTLER, L. The role of subjective theories on love. En: KIEGELMAN (Ed.). Qualitative research in Psychology. Alemania: Schwangau, Huber, 2001.

IÑIGUEZ, L. Investigación y evaluación cualitativa: bases teóricas y conceptuales. Atención Primaria, Barcelona, v. 23, n. 8, p. 496-502, 1999.

KRAUSE, M. Psicoterapia y cambio: una mirada desde la subjetividad. Santiago: Ediciones Universidad Católica de Chile, 2005.

RETUERT, G.; CASTRO, P. Teorias subjetivas de los profesores acerca de su rol profesional en la construcción de la convivencia escolar en establecimientos municipales de enseñanza media de La Serena. Manuscrito en preparación. Universidad de La Serena, 2011.

RODRIGO, M. J.; RODRÍGUEZ, J.; MARRERO, J. Las teorias implícitas: una aproximación al conocimiento cotidiano. Madrid: Visor, 1993.

SEPÚLVEDA, C.; REYES, L.; PEREZ, M. Motivación para el aprendizaje: una mirada desde las aulas chilenas. Santiago: Ediciones UCSH, 2003.

WEINER, B. An Attributional Theory of Motivation and Emotion. New York: SpringerVerlag, 1986a.

. Attribution, Emotion and Action. En: SORRENTINO, R. M.; HIGGINS, E. T. (Eds.). Handbook of motivation and cognition. Foundations of Social Behavior. New York: Guilford Press, 1986b. p. 281-312.

WOOLFOLK, A. Psicología educativa. México: Prentice Hall, 1996.

Texto recebido em 02 de dezembro de 2011.

Texto aprovado em 23 de novembro de 2012. 